\title{
Randomized Concurrent Set Union and Generalized Wake-Up
}

\author{
Siddhartha Jayanti* \\ సిద్ధార్థ జయంతి \\ jayanti@mit.edu \\ MIT
}

\author{
Robert E. Tarjan ${ }^{\dagger}$ \\ prof.tarjan@gmail.com \\ Princeton University \\ Intertrust Technologies
}

\author{
Enric Boix-Adserà \\ eboix@mit.edu \\ MIT
}

\begin{abstract}
We consider the disjoint set union problem in the asynchronous shared memory multiprocessor computation model. We design a randomized algorithm that performs at most $O(\log n)$ work per operation (with high probability), and performs at most $O(m \cdot(\alpha(n, m /(n p))+\log (n p / m+1))$ total work in expectation for a problem instance with $m$ operations on $n$ elements solved by $p$ processes. Our algorithm is the first to have work bounds that grow sublinearly with $p$ against an adversarial scheduler.

We use Jayanti's Wake Up problem and our newly defined Generalized Wake Up problem to prove several lower bounds on concurrent set union. We show an $\Omega(\log \min \{n, p\})$ expected work lower bound on the cost of any single operation on a set union algorithm. This shows that our single-operation upper bound is optimal across all algorithms when $p=n^{\Omega(1)}$. Furthermore, we identify a class of "symmetric algorithms" that captures the complexities of all the known algorithms for the disjoint set union problem, and prove an $\Omega(m \cdot(\alpha(n, m /(n p))+\log (n p / m+1)))$ expected total work lower bound on algorithms of this class, thereby showing that our algorithm has optimal total work complexity for this class. Finally, we prove that any randomized algorithm, symmetric or not, cannot breach an $\Omega(m \cdot(\alpha(n, m / n)+\log \log (n p / m+1)))$ expected total work lower bound.
\end{abstract}

\section{CCS CONCEPTS}

- Theory of computation $\rightarrow$ Concurrent algorithms; Data structures design and analysis; Shared memory algorithms.

\section{KEYWORDS}

disjoint set union, union find, concurrent, asynchronous, data structure, linearizable, wait free, lower bound, Jayanti's wake up

*I am grateful to the Department of Defense (DoD) for their support through the National Defense Science \& Engineering Graduate Fellowship (NDSEG) Program.

$\dagger$ Paritally supported by an innovation research grant from Princeton University and a gift from Microsoft.

¥Supported by a MIT Lemelson Engineering Presidential Fellowship.

Permission to make digital or hard copies of all or part of this work for personal or classroom use is granted without fee provided that copies are not made or distributed for profit or commercial advantage and that copies bear this notice and the full citation on the first page. Copyrights for components of this work owned by others than the author(s) must be honored. Abstracting with credit is permitted. To copy otherwise, or republish, to post on servers or to redistribute to lists, requires prior specific permission and/or a fee. Request permissions from permissions@acm.org.

PODC '19, July 29-August 2, 2019, Toronto, ON, Canada

(C) 2019 Copyright held by the owner/author(s). Publication rights licensed to ACM. ACM ISBN 978-1-4503-6217-7/19/07...\$15.00

https://doi.org/10.1145/3293611.3331593
ACM Reference Format:

Siddhartha Jayanti, Robert E. Tarjan, and Enric Boix-Adserà. 2019. Randomized Concurrent Set Union and Generalized Wake-Up. In 2019 ACM Symposium on Principles of Distributed Computing (PODC '19), July 29-August 2, 2019, Toronto, ON, Canada. ACM, New York, NY, USA, 10 pages. https: //doi.org/10.1145/3293611.3331593

\section{INTRODUCTION}

As data sets get bigger and bigger, it becomes more and more important to harness the potential of parallelism to solve computational problems-even linear time is too slow. In the late twentieth century, many beautiful and efficient algorithms were developed in the PRAM (parallel random access machine) model, which assumes a memory shared among many synchronized processors. In practice, however, synchronization is expensive or may not be possible. A weaker model that has attracted much attention in the distributed systems community is the APRAM (asynchronous parallel random access machine) model, in which a common memory is shared among many unsynchronized processors. In the most general version of this model, any processor can be arbitrarily slow compared to any other.

Obtaining efficiency bounds in the asynchronous model is extremely challenging: the use of locks, for example, seems to make it impossible to guarantee efficiency, since one process could set a lock and then go to sleep indefinitely, blocking progress by any other process that needs access to the same resource. To overcome this problem, systems researchers have invented synchronization primitives that do not use locks, notably CAs (compare and swap) [11], transactional memory [12], and others. These primitives allow at least the possibility of obtaining good efficiency bounds for asynchronous concurrent algorithms. Yet, except for "embarrassingly parallel" computations, this possibility is almost unrealized. Obtaining concurrent algorithms with only small per-operation overheads in the number of processes is a major research goal in distributed data structures. However, efficient algorithms for objects as simple as counters require clever ideas in the face of asynchrony $[6,16]$, and the exact complexities of data structures as fundamental as stacks, queues, and priority queues remain unknown.

An important problem in data structures that could benefit from an efficient concurrent algorithm is disjoint set union, also known as the union-find problem. The simplest version of this problem requires maintaining a collection of disjoint sets, each containing a unique element called its representative, under three operations:

- FIND $(x)$ : return the representative of the set containing element $x$.

- $\operatorname{SameSet}(x, y)$ : return true if $x$ and $y$ are currently in the same set and false otherwise. 
- $\operatorname{Unite}(x, y)$ : if elements $x$ and $y$ are in different sets, unite these sets into a single set and designate some element in the new set to be its representative; otherwise, do nothing.

Initially, each element $x$ is in a singleton set whose representative is $x$. The implementation is free to choose the representative of each new set produced by a unite.

Applications of disjoint set union include storage allocation in compilers [19], finding minimum spanning trees using Kruskal's algorithm [18], maintaining the connected components of an undirected graph under edge additions, testing percolation [21], finding loops and dominators in flow graphs [7], and finding strong components in directed graphs. Some of these applications are generally performed on immense graphs, notably finding connected components and finding strong components [3, 4, 21]. In particular, model checking requires finding strong components in huge, implicitly defined directed graphs [3, 4]. There are sequential lineartime strong components algorithms [22], but the model-checking application could potentially benefit from an even faster algorithm. The sequential algorithms use depth-first search [22], which apparently cannot be efficiently parallelized [20]. If one had an efficient concurrent disjoint set union algorithm one could use it in combination with breadth-first search to potentially speed up model checking. This application, described to the second author by Leslie Lamport, was the original motivation for our work.

The classical sequential algorithm for the disjoint set union problem is the compressed tree data structure [23, 24]. With appropriate tree linking and path compaction rules, $m$ operations on sets containing a total of $n$ elements take $O(m \cdot \alpha(n, m / n))$ time [23, 24], where $\alpha$ is a functional inverse of Ackermann's function, defined in Section 3. Three linking rules that suffice are linking by size [23], linking by rank [24], and linking by random index [10]; three compaction rules that suffice are compression [23], splitting [24], and halving [24]. The set union algorithm obtained by combining any of these linking and compaction rules has asymptotically optimal amortized time complexity for the sequential problem; this was proved by Fredman and Saks [8].

There have been two significant works on the concurrent set union problem. While both works have significant ideas that have propelled our work, they contain flaws that reveal the subtlety of the problem and the difficulty in analyzing even simple tree data structure manipulations in a concurrent shared memory system.

The goal of designing a concurrent set union algorithm is to prove an amortized per-operation work bound that is as close to the sequential work bound as possible. The amortized work bounds for all the algorithms we discuss in this paper are of the form $O(\alpha(n, \cdot)+\Delta(p))$. Here, the inverse-Ackerman term, $\alpha(n, \cdot)$, is nearly equal to the $\alpha\left(n, \frac{m}{n}\right)$ term that is optimal for the sequential case. The extra additive term $\Delta(p)$ is called the concurrency overhead, since it represents the extra work per operation that is needed by the data structure in order to support concurrent access by the $p$ processes. Since the inverse-Ackermann term is almost constant, a single process running an optimal sequential set union algorithm can do $\Theta\left(1 / \alpha\left(n, \frac{m}{n}\right)\right) \approx \Theta(1)$ operations in a unit of time. In the concurrent case, the work complexity of the data structure is approximately proportional to $\Delta(p)$ if we ignore inverse-Ackermann terms. Thus, if $p$ processes are using the data structure together, and we consider the ideal case where they each run at approximately the same speed as a single uniprocessor, they can perform about $\Theta(p / \Delta(p))$ operations in unit time. So, we define $p / \Delta(p)$ as the speed-up of a concurrent algorithm, and hope to use algorithms where $\Delta(p)$ is at most polylogarithmic to get near-linear speed-ups that facilitate the possibility of fast parallel algorithms. (For some algorithms the function $\Delta(\cdot)$ can also depend on $m$ and $n$ for technical reasons; we have simply omitted these parameters here to emphasize the semantic meaning of the term.)

Anderson and Woll were the first to define and attempt the concurrent version of the set union problem [1]. They set up a framework to facilitate turning compressed tree sequential algorithms into concurrent algorithms, and developed a concurrent solution to the set union problem that combines linking by rank with a concurrent version of path halving. In their problem formulation, they note that many applications of the set union data structure benefit from having a $\operatorname{SAMESET}(x, y)$ operation that returns whether $x$ and $y$ are in the same set. A highlight of their work was a succinct algorithm that reduces SAMESET to FIND while cleverly avoiding possible errors due to concurrency. Both Jayanti and Tarjan's algorithm (discussed next) and our present work use Anderson and Woll's original SAMESET algorithm, whose pseudo-code is in Appendix A. The amortized and worst-case work complexities of SAMESET are identical to those of UNITE in the case of each of the algorithms. Anderson and Woll claimed an amortized $\Theta(\alpha(m, 1)+p)$ work bound, corresponding to a linear concurrency overhead of $\Delta(p)=p$. Unfortunately, the proof of their upper bound is incomplete, since they try to reduce to the sequential algorithm's proof without accounting for interference among different processes doing halving on intersecting paths. Their analysis describes in detail how their algorithm can create equal rank chains of length $\Omega(p)$, and thus how their algorithm can require $\Omega(n p)$ work to do $n-1$ unite operations. Thus in the worst case their algorithm gives no speed-up. This sparked further research into the problem.

While Anderson and Woll attempted to parallelize the deterministic compressed trees algorithm that links by rank and does compaction by path halving, Jayanti and Tarjan instead chose to work on linking by random index and compaction by splitting [17]. The change in linking method is key to their work. In their algorithm, the $n$ nodes of the set union object are given randomized indices, i.e. a random permutation of the natural numbers from 1 through $n$, in the preprocessing phase. They assume that the adversary does not know this random permutation. They use the simple linking rule that links a node of a smaller index under a node of a larger index. Jayanti and Tarjan do not analyze their algorithm under an adversarial scheduler. Rather, they define a particular type of scheduler that guarantees that the linearization order of set union operations performed by the processes is independent of the schedule. Unfortunately, even an oblivious scheduler, or more simply a scheduler that gives processes turns in rounds, does not necessarily satisfy this property. They claimed a work bound of $O(m$. $(\alpha(n, m /(n p))+\log (n p / m+1)))$ if the schedule is of the correct type, which would reduce $\Delta(p)$ to $\log (p)$ for this class of schedules. Unfortunately their attempt to account for interference during concurrent splits has a subtle error. Correcting it results in the slightly worse bound of $O\left(m\left(\alpha\left(n, m /\left(n p^{2}\right)\right)+\log \left(n p^{2} / m+1\right)\right)\right)$, that still gives $\Delta(p)=\log (p)$. We believe this bound is tight for 
their algorithm. If the scheduler is adversarial, however, there are sequences of $O(n)$ operations that take $\Omega\left(n p^{1 / 2}\right)$ expected work, making the speed-up for this algorithm at best the square root of the number of processes.

In this paper, we develop the first algorithm for union-find that provably gives speed-up under adversarial scheduling. In fact, our algorithm gives near-linear speed-up. An initial hope would be to design an algorithm with no concurrency overhead (i.e. $\Delta(p) \equiv 0$ ). However, we prove that this is impossible by giving many shared memory step complexity lower bounds for the set union problem. A main contribution of our paper is a proof that $\Delta(p)=\Omega\left(\log \log \frac{n p}{m}\right)$. Furthermore, we give compelling evidence that an even stronger lower bound may hold for the problem, by showing that there is an $\Omega(\log \min \{n, p\}+\log (n) / \log \log (n))$ lower bound on worst-case work complexity, and for the large class of symmetric algorithms-a class that includes all known set union algorithms and techniques to parallelize sequential algorithms a stronger $\Omega(\alpha(n, m /(n p))+$ $\log (n p / m+1))$ lower bound on amortized work complexity. Thus, the goal is to design an algorithm with $\Delta(p)=\log (n p / m)$. A highlight of our paper is the design and analysis of a randomized symmetric algorithm that has an expected work complexity of $\Theta(\alpha(n, m /(n p))+\log (n p / m+1))$, matching the lower bound. Furthermore, with high probability the worst-case work complexity of a single set union operation for the algorithm is $O(\log n)$, which is optimal if $p=n^{\varepsilon}$ for any constant $\varepsilon$ and at most a factor of $\log \log (n)$ away from optimality regardless of $p$. A sequential algorithm of Blum shows that set union operations can be done in at most $O(\log (n) / \log \log (n))$ if $p=1$ [5].

Our lower bound proofs introduce a novel technique, which we call the generalized wake-up technique, for proving lower bounds on the amortized complexities of operations on linearizable data types that are built from primitive variables supporting read, write, and compare-and-swap (CAS). Our technique is developed by generalizing Jayanti's wake-up problem, and extending his lower bound analysis to our generalized version of the problem [15]. As we show in the paper, our technique is general and gives amortized lower bounds on the complexities of several other data types along with set union. In particular, we also obtain the following two results:

(1) Define an $r$-relaxed counter as a fetch-and-increment object whose "fetch" returns a value that is within an additive $r$ of the correct counter value. Then $m$ operations on a ( $1-$ $\varepsilon) p / 2)$-relaxed counter take $\Omega(m \log p)$ steps in the worst case.

Remark: Our lower bound shows that Ellen and Woelfel's implementation of fetch-and-increment [6] and Jayanti's implementation of a counter [16] have optimal amortized step complexity. Furthermore, the $\Omega(\log p)$ overhead for counting in an asynchronous system holds even for randomized algorithms, and even if the counter needs to be only approximately accurate.

(2) Define an $r$-relaxed priority queue as an object that returns one of the top $r$ items in priority order. For $r>1$, this is a relaxation of the standard priority queue-which corresponds to the 1-relaxed priority queue. Then $m$ deleteMin operations on a $((1-\varepsilon) p)$-relaxed priority queue take $\Omega(m \log p)$ steps in the worst case. We also prove analagous results for the $r$-relaxed stack and $r$-relaxed queue objects.

The remainder of our paper consists of four sections and the appendices. Section 2 contains a description of the shared-memory model. Section 3 motivates and defines the generalized wake up problem, and proves our lower bounds. Section 4 presents our algorithm for the set union problem. We end with some remarks and open problems in Section 5.

\section{CONCURRENCY MODEL}

We consider the shared memory asynchronous multiprocessor model of computing with $p$ processes $P=\pi_{1}, \ldots, \pi_{p}$ that run concurrently. We assume that each process $\pi_{i}$ has access to both a (small) private memory and the collective shared memory. We assume that the size of shared memory words is $O(\log n)$ bits, where $n$ is the number of nodes in the data structure. Asynchronicity is modeled by an adversarial scheduler. We call a process active if it is currently executing instructions. At each time step $t$ of the asynchronous system, the scheduler gets to adversarially pick which active process it would like to allocate the next step to. This decision can be based on the current state of shared memory, process program counters, and past randomness, but not on future randomness. The resultant time indexed sequence $\sigma$ of which process got to execute at which time steps is called the schedule. The history of a process $\pi_{i}$ up to time $t$ is the entire sequence of operations performed by $\pi_{i}$ and return values received by $\pi_{i}$ up to time $t$. In particular, if the history of a particular process $\pi_{i}$ is the same if $t_{1}$ steps of algorithm $A$ are executed under schedule $\sigma_{1}$ or if $t_{2}$ steps of algorithm $A$ are executed under schedule $\sigma_{2}$, then the two scenarios are indistinguishable to $\pi_{i}$. The notion of indistinguishability is key to impossibility results.

To provide synchronization of writes to shared memory, we use the atomic compare and swap primitive CAs $(x, a, b)$. Given the address $x$ of a word in shared memory and two values $a$ and $b$, the CAs operation tests whether word $x$ holds value $a$; if so, it stores value $b$ in word $x$ (overwriting $a$ ) and returns true; if not, it returns false. In general, we allow values written to shared memory via a regular write or CAs operation to include random bits. We use $\$$ to symbolize a random bit in our pseudo-code.

We are interested in concurrent algorithms for set union that are linearizable [13] and bounded wait-free [11, 13]. To be linearizable means that (i) the outcome of a concurrent execution is the same as if each set operation were executed instantaneously at some distinct time (its linearization point) during its actual execution and (ii) the sequential execution sequence given by the linearization points is correct; that is, all find operations produce answers that are correct at their linearization points. The linearization points define a total order of the operations, called the linearization order. To be bounded wait-free means that every operation finishes in a bounded number of its own steps.

We measure the efficiency of algorithms using two different but very related metrics. The work complexity, or simply work, done by a concurrent algorithm is the total number of primitive instructions done by all processes to complete all operations. The step complexity is defined almost identically, but only counts shared 
memory instructions. The amortized work of an algorithm is its total work divided by the total number of operations started across all processes. The amortized step complexity is defined analogously. Since the work complexity is always bounded below by the step complexity, upper bounds on work complexities and lower bounds on step complexities are the strongest results. When analyzing our algorithms, we will use the convention that $m$ is the number of operations, $n$ is the number of nodes, and $p$ is the number of processes. In general, we assume that $m \geq n / 2$-every node is part of some operation, and $m \geq p$-there is at least one operation per process.

We call an algorithm symmetric if it satisfies the following three properties: (1) data structure operations have the same code for all processes, i.e. the code does not depend on process IDs; (2) all random bits used by a process are written directly to shared memory via a write or CAS; (3) the algorithm does not use the (true/false) return values of CAS operations. This class of algorithms is of particular interest to us, since it captures the complexity of all known algorithms for the set union problem in the following sense. The algorithms of Anderson and Woll and Jayanti and Tarjan superficially break from (3) by using the return value of the CAs operation in the link step; however, it is straightforward to verify that simply removing the return value of the CAS (i.e., making all CAS operations return false even if they succeed), affects neither correctness nor any work complexity bounds for their algorithms. Our algorithm presented in Section 4 is symmetric as presented.

\section{LOWER BOUNDS}

In this section we prove three step complexity lower bounds on the set union object: a worst-case operation step complexity bound that holds for all algorithms; an amortized operation step complexity bound that holds for symmetric algorithms; and a weaker amortized operation step complexity bound that holds for all algorithms. In subsequent sections, we will design and analyze a symmetric algorithm that matches the first two lower bounds.

Our proofs depend on the previous results of Jayanti [15] and Fredman and Saks [8]. We briefly recapitulate these results below.

\subsection{Preliminaries}

In the asynchronous scheduling model, no process knows when it and other processes will be given their first step. fayanti's wake-up problem [15], requires one of the $p$ processes to discover that all processes have been given at least one step. Formally, the problem is to give code for the $p$ processes such that the following conditions are met:

(1) (termination) Each process $\pi_{i}$ returns either false or true in a bounded number of its own steps.

(2) (truthfulness) No process $\pi_{i}$ returns a true before all processes have taken at least one step.

(3) (non-triviality) At least one process must return true.

Theorem 3.1 (Jayanti's Theorem: Theorem 6.1 in [15]). Let $\mathcal{A}$ be a randomized algorithm that solves fayanti's wake-up for the processes in set $P$ of size $p=|P|$. Then, there is a schedule $\sigma$, such that the processes of $P$ executing $\mathcal{A}$ under schedule $\sigma$ results in some process performing $\Omega(\log p)$ steps in expectation.
Fredman and Saks show a tight cell probe lower bound on the deterministic complexity of the sequential set-union problem. Their theorem extends to randomized algorithms to yield the following theorem.

Theorem 3.2 ([8]). Let $\mathcal{A}$ be any deterministic or randomized algorithm that solves the sequential set-union problem for $n$ nodes. (1) The expected amortized work complexity of $m \geq n$ operations is $\Omega(\alpha(n, m / n))$, and (2) the expected worst-case work complexity of a single operation is $\Omega(\log (n) / \log \log (n))$.

\subsection{Concurrent Set Union Lower Bounds from Jayanti's Wake Up}

We solve Jayanti's wake up via set union to get a lower bound on the complexity of a single operation.

Lemмa 3.3. Algorithm 1 solves the wake-up problem for $k$ processes using a disjoint set union instance with $k+1$ nodes, where each process executes one UNITE and two FIND operations.

Proof. Let $q_{1}, \ldots, q_{k}$ be the $k$ processes and let the nodes be labelled $0, \ldots, k$. The algorithm below correctly solves wake-up because:

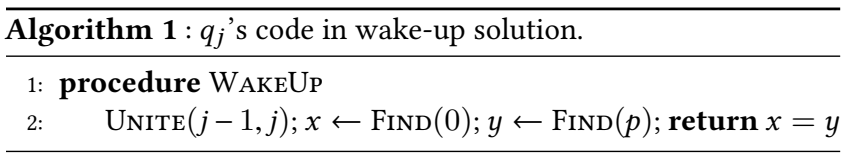

(i) the last process to complete UNITE finds that the representatives of nodes 0 and $k$ are the same and thus returns true, and (ii) no process returns true before all processes have linearized their UNITE operations, since no representative of $k$ can be the same as any representative of 0 until all of the nodes are in the same set.

THeOREм 3.4. Let $\mathcal{A}$ be any linearizable wait-free algorithm for the set union problem on $n$ nodes with $p$ processes. The expected work complexity of a single operation with $\mathcal{A}$ is $\Omega(\log \min \{n, p\}+\log (n) / \log \log (n))$.

Proof. Instantiate Lemma 3.3 with $k=\min \{n-1, p\}$. The most expensive of the three set union operations of the process that performs the most work in the adversarial schedule of Theorem 3.1 must do $\Omega(\log \min \{n, p\})$ expected work. Combining this with the worst-case lower bound from Theorem 3.2 yields the theorem.

We extend the worst-case lower bound to an amortized lower bound for symmetric algorithms. The key ideas in this extension are the use of a shadowing schedule and a combinatorial argument.

Lemma 3.5. Let $\mathcal{A}$ be any symmetric linearizable wait-free algorithm for the set-union problem on $n$ nodes and $p$ processes. The amortized work of $m$ operations is at least $\Omega(\log (n p / m+1))$ in expectation.

Proof. Divide the $n$ nodes into $g=\frac{m}{p}$ groups of size $k+1$, where $k=\frac{n p}{8 m}$ (disregard any additional nodes); label the groups $G_{1}, \ldots, G_{g}$. Note that $m \geq p$ and $m \geq \frac{n}{2}$, so $k \leq \frac{p}{4}$. We divide $\frac{p}{2}$ processes into two sets $A=\left\{q_{1}, \ldots, q_{k}\right\}$ and $B=\left\{q_{k+1}, \ldots, q_{p / 2}\right\}$. Note that $|B| \geq \frac{p}{4}$ and $|A \cup B|=\frac{p}{2}$. 
Consider running the wake-up algorithm of Lemma 3.3 on the $k$ processes in $A$ using the $k+1$ nodes in $G_{1}$. By Theorem 3.1 there is a schedule $\sigma_{1}$ in which some process $q_{i}$ performs $\Omega(\log k)$ steps. Assign to each process in $B$ the same sequence of three set union operations as what $q_{i}$ performs, and define schedule $\sigma_{1}^{\prime}$ to be the schedule $\sigma_{1}$, with the processes of $B$ interleaved to run in lockstep with $q_{i}$. In this schedule, the processes $q_{1}, \ldots, q_{p / 2}$ perform $\Omega(p \log k)$ work to do $\frac{3}{2} p$ set union operations. Repeating this procedure on each group $G_{j}$ produces schedules $\sigma_{j}^{\prime}$, each of which performs $\Omega(p \log k)$ work. Therefore, in the concatenated schedule of $\sigma_{1}^{\prime} \sigma_{2}^{\prime} \cdots \sigma_{g}^{\prime}$, the processes $q_{1}, \ldots, q_{p / 2}$ perform a total of $\Omega(g p \log (k+1))=\Omega(m \cdot \log (n p / m+1))$ work to do a total of $g p=m$ operations.

The lower bound from Lemma 3.5 becomes trivial when $m \geq n p$. The next lemma shows that the amortized work complexity is still lower bounded by an inverse-Ackermann term.

Lemma 3.6. Let $\mathcal{A}$ be any symmetric linearizable wait-free algorithm for the set-union problem on $n$ nodes and $p$ processes. The amortized work of $m \geq n$ operations is at least $\Omega(\alpha(n, m /(n p))$ in expectation.

Proof. The main idea of the proof is to use a shadowing schedule on the result of Theorem 3.2. We break the proof into two cases, $m>n p$ and $n \leq m \leq n p$.

Case $m>n p$ : In this case, we let $M=m / p$. Since $M>n$, Theorem 3.2 yields a sequence of $M$ operations that can be performed on a single process resulting in $\Omega(M \cdot \alpha(n, M / n))=\Omega\left(\frac{m}{p}\right.$. $\alpha(n, m /(n p)))$ work. Simply give each of the $p$ processes this same sequence of operations. Running the processes in a lock-step schedule as in Lemma 3.5 will make each of them do the same amount of work since the algorithm is symmetric. So, the total work is $\Omega(m \cdot \alpha(n, m /(n p)))$.

Case $n \leq m \leq n p$ : In this case, $m /(n p)$ is just a constant between 0 and 1 , so it suffices to show a sequence of $m$ operations that requires $\Omega(m \cdot \alpha(n, c))$ for any constant $c$, since all of these values are within constant factors of each other. So, let $p^{\prime} \geq 1$ be a number of processes such that $n\left(p^{\prime}+1\right)>m>n p^{\prime}$. Repeating the argument from the $m>n p$ case using only $p^{\prime}$ processes instead of all $p$ yields a lower bound of $\Omega\left(m \cdot \alpha\left(n, m /\left(n p^{\prime}\right)\right)\right)=\Omega(m \cdot \alpha(n, c))$ where $c \approx 1$.

Theorem 3.7. Let $\mathcal{A}$ be any symmetric linearizable wait-free algorithm for the set-union problem on $n$ nodes and $p$ processes. The amortized work of $m$ operations is at least $\Omega(\alpha(n, m /(n p))+\log (n p / m+$ 1)) in expectation.

Proof. Combining the lower bound results of Lemmas 3.5 and 3.6 yields the theorem.

The lower bound of Theorem 3.7 is tight, as we will show by designing a symmetric algorithm whose work complexity matches the step complexity lower bound. However, it still leaves open the question of whether there is a fundamental barrier to eliminating concurrency overhead for non-symmetric algorithms. We will now develop a new technique, called the generalized wake up technique, for showing amortized lower bounds that will imply an
$\Omega\left(\log \log \frac{n p}{m}\right)$ lower bound on the amortized complexity of shared memory set union operations.

\subsection{Generalized Wake Up}

To motivate generalized wake up, we consider the reduction in Algorithm 1 again. We were able to get a worst case lower bound on the step complexity of set union using the reduction, since Jayanti's theorem showed a non-trivial lower bound on the step complexity of a single process in any solution to his wake up problem. If however, we could show a non-trivial lower bound on the total step complexity (over all processes) for a solution to Jayanti's wake-up, then we could get an amortized lower bound on the step complexity of set union using the same reduction. Unfortunately, this is not possible since Jayanti's wake-up problem can be solved in $O(p)$ total work. But we dig deeper into the proof idea of Jayanti's Theorem to reveal that generalizing the wake-up problem can lead to a different problem that is strong enough to show powerful amortized lower bounds. We give an informal description of this proof method below.

Consider the setting where the processes in the set $P$ are running a randomized algorithm $\mathcal{A}$ that uses variables in the set $V$. At any point of time, we can define a knowledge set for each process $\pi_{i}$. The knowledge set of $\pi_{i}$ at time $t$ is the set of processes that $\pi_{i}$ can infer are awake, i.e. have taken at least one step, based on its history-its sequence of operations on shared memory and the values it received in return. Similarly, we can define a knowledge set for each variable $x \in V$. The knowledge set of $x$ is the set of processes that a process could infer is awake if it reads $x$. Since a process can return true only after it is sure that every other process is awake, the process that returns 1 must have a knowledge set of size $p$ before it returns. Jayanti proves his theorem by constructing a special schedule $\sigma^{*}$ and a sequence of "knowledge" functions parametrized by round $r: K_{r}:(P \cup V) \rightarrow 2^{P}$, and shows that:

(1) According to $\sigma^{*}$, each process that is yet to return takes exactly one step in each round $r$.

(2) $\left|K_{r}(\Psi)\right|$ is an upper bound on the knowledge set of entity $\Psi \in(P \cup V)$ after round $r$.

(3) $\left|K_{r}(\Psi)\right| \leq 4^{r}$ for any round $r$.

By the last inequality, Jayanti shows that when the algorithm $\mathcal{A}$ is run under schedule $\sigma^{*}$, there must be at least one process that does not return until round $r=\log _{4} p$.

In order to get the correct generalization of the wake-up problem, we interpret the above schema in the following way.

OBSERVATION 3.1. The step complexity lower bound for wake-up comes about because a single process must reveal that it has a large knowledge set.

Therefore, in order to get a stronger lower bound, we define a generalization of wake-up that forces multiple processes to reveal that they have large knowledge sets. Thus motivated, we define the generalized wake-up problem below.

The Generalized Wake-Up (GWU) family of problems for $p$ processes is parametrized by a monotonically non-increasing sequence $s_{1}, \ldots, s_{p}$ of values between 1 and $p$. The problem $G W U\left(s_{1}, \ldots, s_{p}\right)$ is to design an algorithm for the $p$ processes such that each process $\pi_{i}$ returns an integer in the range $[1, p]$ (a lower bound on the the 
number of processes it knows to have woken up), that satisfies the following conditions:

(1) (termination) Each process $\pi_{i}$ must return a value in the range $[1, p]$.

(2) (truthfulness) If $\pi_{i}$ returns $k$ at time $t$, then at least $k$ processes are awake.

(3) (non-triviality) At least $k$ processes must return a value greater than $s_{k}$.

Informally, generalized wake-up specifies a sequence of values, and the algorithm must ensure that the processes demonstrate at least as much knowledge as the sequence requests.

Observe that Jayanti's wake-up problem is the instance $G W U(p, 1,1, \ldots, 1)$. Also, note that there is no algorithm with bounded step complexity for instances of generalized wake-up where $s_{i}>p-i+1$, since the scheduler can wait for an unbounded amount of time before letting the $(p-i) t h$ process wake up. So, we call the instance $G W U(p, p-1, p-2, \ldots, 1)$ the Strong Wake$U p(S W U)$ problem, and in general require that the sequence $s$ be majorized by the sequence of descending numbers from $p$.

The following is our main theorem about Generalized Wake-Up; a formal proof of this theorem will appear in the full version of this paper due to space constraints in the abstract.

TheOREM 3.8. Let $\mathcal{A}$ be a randomized algorithm that solves the problem $G W U\left(s_{1}, \ldots, s_{p}\right)$ for processes $\pi_{1}, \ldots, \pi_{p}$. There is an asynchronous schedule $\sigma$ such that when algorithm $\mathcal{A}$ is run under schedule $\sigma$, the total number of steps performed by all the processes is $\Omega\left(p+\sum_{i=1}^{p}\left(\log s_{i}\right)\right)$.

While it relies on a simple observation, Theorem 3.8 yields powerful amortized lower bounds for some data structures. In the next section, we prove an such a lower bound for set union. Please see Appendix B for more results.

\subsection{Concurrent Set Union Lower Bound from Generalized Wake Up}

In order to prove an amortized lower bound on set union operations complexities, we find a set union instance that is weak enough to be solvable by set union but also strong enough to be provably hard by Theorem 3.8. The key therefore is to design a set union based algorithm that ensures that many processes gain knowledge of many other processes being awake even in the presence of an adversary that will schedule the processes in a way that minimizes process knowledges given any fixed algorithm. Our high-level idea for forcing processes to gain knowledge is to leverage path lengths in graphs. We start with an empty graph on the vertex set $V$, and a fixed set $E \subseteq V \times V$ of edges to be added (generally not including all possible edges). A process $p$ can dynamically "add the edge $(u, v) \in E$ " to the graph by performing $\operatorname{Unite}(u, v)$. After some processes have added some edges, there is a resultant graph $H=$ $(V, F)$, where $F \subseteq E$ is the set of edges that have already been added to the graph. A process $p^{\prime}$ can "check if vertices $u^{\prime}$ and $v^{\prime}$ are connected" in the resultant graph $H$ by performing $\operatorname{SAMESET}\left(u^{\prime}, v^{\prime}\right)$. We observe that if $\operatorname{SAmESET}\left(u^{\prime}, v^{\prime}\right)$ is true, then at least $\delta\left(u^{\prime}, v^{\prime}\right)$ edges must have been added to the graph, where $\delta$ is the shortest path length function on the original graph $G=(V, E)$. In particular, if each process is somehow restricted to add at most one edge in our algorithm, then a process that sees that $\operatorname{SAMESET}\left(u^{\prime}, v^{\prime}\right)$ is true learns that at least $\delta\left(u^{\prime}, v^{\prime}\right)$ processes are awake. On the other hand, if $\operatorname{SAmESET}\left(u^{\prime}, v^{\prime}\right)$ is false, no wake-up knowledge is gained. So, in order to get an amortized bound, we must design a procedure to ensure that many processes will get non-trivial wake-up information, so that we can leverage the generalized wake-up lower bound.

We will see as the proof unfolds that ensuring many SAMESET operations return true amounts to picking a highly connected graph $G$. However, the length of the shortest paths become smaller as connectivity goes up. For instance, in the best connected graphthe complete graph-all shortest paths are of length one, and thus even when SAMESET operations return true, very little wake-up information is gained. This inherent trade-off between connectivity and long paths makes the graph we pick pivotal to the proof. We formalize the notion of graph connectivity that we will require:

Definition 3.9. The edge expansion constant $h(G)$ of a graph $G=(V, E)$ is defined as

$$
h(G) \triangleq \min _{\substack{S \subset V \\|S| \leq|V| / 2}} \frac{\left|E_{\text {out }}(S)\right|}{|S|},
$$

where $E_{\text {out }}(S)$ is the set of edges that have one vertex in $S$ and one in $V-S$.

The edge expansion constant measures the connectivity of a graph-the higher the constant, the more connected the graph is. Sparse graphs with a high edge expansion constant are called expanders, and are known to have many applications in computer science [14]. The following result implied by Friedman [9] states that good expanders exist for any number of vertices. These are the graphs that we will use in our proof:

LEMMA 3.10 (By Friedman [9]). For every large-enough $n$, there is a 4-regular expander graph $\mathcal{G}_{n}=(V, E)$ with $|V|=n,|E|=2 n$, and $h\left(\mathcal{G}_{n}\right)>0.2$.

Moreover, all subgraphs of an expander with sufficiently many edges have large connected components:

Lemma 3.11. Let $a=0.95<1$ and $b=0.5>0$. Then for large enough $n$, if $H=(V, F)$ is a subgraph of $\mathcal{G}_{n}=(V, E)$ with $|F| \geq a|E|$, then the largest connected component of $H$ has size at least $b|V|$.

Proof. Assume by contradiction that all connected components $C_{1}, \ldots, C_{\ell}$ of $H$ have size less than $|V| / 2$. Then, by definition of $h(\cdot)$, there are at least $h\left(\mathcal{G}_{n}\right) \cdot\left|C_{i}\right|$ edges of $E$ between $C_{i}$ and $V-C_{i}$. Thus,

$|E|-|F| \geq \frac{1}{2} \sum_{i=1}^{\ell}\left|E_{\text {out }}\left(C_{i}\right)\right| \geq \frac{1}{2} \sum_{i=1}^{\ell} h(G)\left|C_{i}\right|=\frac{h(G)|V|}{2}=\frac{h(G)|E|}{4}$.
So $|F| \leq|E|(1-h(G) / 4)<0.95|E|$.

We will now show how to solve a non-trivially hard instance of generalized wake up via set union operations. For any integer $t>0$ and large enough $n$ so that $\mathcal{G}_{n}$ has the properties of Lemma 3.10 and Lemma 3.11, let $p=2 n^{3} t$ and $m=2 p$. We describe an algorithm on $p$ processes that performs $m$ operations of concurrent disjoint set union on $n$ nodes. 
Work with $\mathcal{G}_{n}=(V, E)$. Define a process type to be a triple $(e=$ $(u, v), x, y)$ where $e \in E$ and $u, v, x, y \in V$. Since $|E|=2|V|=2 n$, there are a total of $2 n^{3}=p / t$ process types, so we can create $t$ processes of each type. The process of type $((u, v), x, y)$ performs the steps in Algorithm 2. (Note that $\delta(x, y)$ is a constant known to the process ahead of time.)

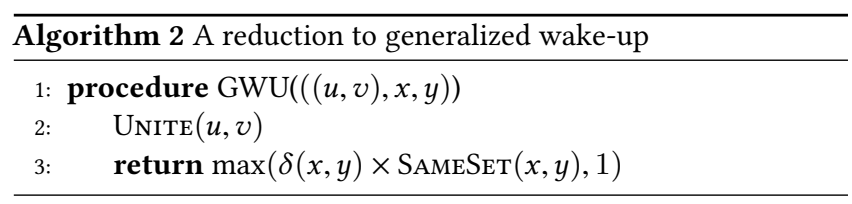

Order the processes as $\pi_{1}, \ldots, \pi_{p}$ based on the linearization points of completing the UNITE operation on line 2. We denote the type of $\pi_{i}$ by $T\left(\pi_{i}\right)=\left(e_{i}=\left(u_{i}, v_{i}\right), x_{i}, y_{i}\right)$. Let $I=[1, \ell]$, be the indices of the first $\ell$ processes to linearize their UNITE operations; and define $H=(V, F)$ where $F=\bigcup_{i \in I}\left\{e_{i}\right\}$ is the set of edges. The constant $\ell$ will be defined by the schedule to be the smallest constant that ensures a "large" connected component in $H$ by Lemma 3.11. In particular, pick $\ell$ to be the minimum number such that $|F| \geq a|E|$. Let $C$ be the set of vertices in the largest connected component of $H$. Let $P \triangleq\left\{\pi_{i}: e_{i} \notin F\right\}$ be the set of processes whose UNITE operation in Algorithm 2 will not attempt to add an edge in $F$. Finally, let $a, b$ be the constants from Lemma 3.11 .

We will now prove a series of short lemmas that have the following informal meanings:

(1) The largest connected component in $H=(V, F)$, i.e. $C$, contains a constant fraction of the vertices in $V$. Furthermore, the processes in $P$ which will attempt to unite edges not in $F$, is at least a constant fraction of the total number of processes.

(2) If $x$ and $y$ are random vertices in $V$, then with constant probability they will be in the connected component $C$ and the distance between them will be $\Omega(\log n)$.

(3) The first two lemmas imply that a constant fraction of the processes will return a value that is $\Omega(\log n)$.

(4) Theorem 3.8 then implies the $\Omega(\log \log n)$ lower bound.

LemMA 3.12. $|C| \geq b n=\Omega(n)$ and $|P| \geq(1-a) p-p /|E|=$ $\Omega(p)$.

Proof. $|C| \geq b|V|=b n$ by Lemma 3.11, since $|F| \geq a|E|$.

Since there are $p /|E|$ processes that are trying to perform a union involving the vertices of any given edge in $G,|P| \geq(|E|-\lceil a|E|\rceil)$. $\frac{p}{|E|} \geq p(1-a)-p /|E|$.

Lemma 3.13. Let $(x, y)$ be a random pair of vertices from $V$.

$\mathbb{P}\left(x, y \in C\right.$ and $\left.\delta(x, y)>(1 / 2) \log _{4} n\right) \geq b^{2}(1-2 /(b \sqrt{n}))$.

Proof. $\mathbb{P}(x, y \in C) \geq b^{2}$ because $|C| \geq b|V|$ by Lemma 3.12 . And $\mathbb{P}\left(\delta(x, y)>(1 / 2) \log _{4} n \mid x, y \in C\right)>1-2 /(b \sqrt{n})$, since by the 4-regularity of $\mathcal{G}_{n}$, the radius $r>1$ neighborhood of vertex $x$ has size at most $2 \cdot 4^{r}$. So, the $r=(1 / 2) \log _{4} n$ neighborhood of $x$ has at most $2 \sqrt{n}$ vertices.

Lemma 3.14. A constant fraction of the processes return a value that is $\Omega(\log n)$.
Proof. All the processes $\pi_{i} \in P$ for which $x_{i}, y_{i} \in C$ and $\delta\left(x_{i}, y_{i}\right) \geq$ $(1 / 2) \log _{d} n$, return at least $(1 / 2) \log _{d} n=\Omega(\log n)=\Omega(\log p)$, since $i>\ell$ by the definition of $P$. By Lemma 3.13, the fraction of such processes returning $\Omega(\log n)$ is at least $b^{2}(1-2 / b \sqrt{n})$. So the total number of such processes is at least $|P| b^{2}(1-2 / b \sqrt{n}) \geq \Omega(p)$, by Lemma 3.12 .

LeMma 3.15. Algorithm 2 solves $G W U\left(s_{1}, \ldots, s_{p}\right)$ where $s_{1}=$ $s_{2}=\cdots=s_{\kappa}=\Omega(\log n), s_{\kappa+1}=\cdots=s_{p}=1$ and $\kappa=\Omega(p)$.

Proof. We argue each of the generalized wake up conditions. Termination follows by wait-freedom of the Union Find data structure. Truthfulness follows since every process adds at most a single edge to the graph through its unite operation on line 2, so a successful $\operatorname{SAmeSet}(x, y)$ operation indicates that at least $\min (1, \delta(x, y))$ processes have woken up. And non-triviality follows by Lemma 3.14

We now translate the lower bound from Lemma 3.15 into a lower bound parametrized by the number of operations $m$, the number of processes $p$, and the size of the set union object $n$, through a combinatorial argument.

THEOREM 3.16. Consider any randomized algorithm for p-process shared memory set union on an instance with $n$ nodes. Let $m>n$ and $m>2 p$. Then, there is a sequence of $m$ set union operations on the $p$ processes and $n$ nodes that takes $\Omega(m \log \log (n p / m))$ shared memory steps.

Proof. Divide the $n$ nodes into groups of size $s=\lfloor 2 n p / m\rfloor$. Have $g=\lfloor m / 2 p\rfloor$ groups $V_{1}, \ldots, V_{g}$, ignoring extra nodes.

For each group $V_{i}$, do not use all the nodes in the group, but only use $s^{\prime}=\Theta\left(s^{1 / 3}\right)=O\left(p^{1 / 3}\right)$ of the nodes, so that we can choose $t$ so that $p^{\prime} \triangleq 2\left(s^{\prime}\right)^{3} t=\Omega(p)$ and $p^{\prime} \leq p$, and can run Algorithm 2 on $p^{\prime}$ processes, where there are $t$ processes of each type. By Lemma 3.15 and the GWU lower bound (Theorem 3.8), running Algorithm 2 with these parameters takes $\Omega\left(p^{\prime} \log \log s^{\prime}\right)=$ $\Omega(p \log \log (n p / m))$ shared memory operations in the worst case.

We therefore run Algorithm 2 as above for each group of nodes, in a series of $g$ rounds, letting all operations from the previous round complete before starting the next one. The total worst-case cost is $\Omega(g p \log \log (n p / m))=\Omega(m \log \log (n p / m))$, the total number of processes used is $\leq p$, the total number of nodes used is at most $n$, and the total number of set union operations invoked is at most $2 p^{\prime} \cdot g \leq 2 p \cdot m /(2 p)=m$.

In the remainder of the paper, we describe our randomized algorithm for set union.

\section{OUR CONCURRENT ALGORITHM}

Our concurrent set union algorithms use the same structure as the best sequential algorithms: a compressed forest. This forest contains one rooted tree per set, whose nodes are the elements of the set and whose root is the set representative. We assume that the nodes have indices 1 through $n$ and thus can be compared via ' $<$ '. Each node $x$ has a field $x . p$ to store the address of a parent node, a field $x . r$ to store a non-zero integer rank, and a field $x . b$ to store a single root-bit signifying whether or not $x$ is the root of its tree. Initially, $x . p=x, x . r=0$, and $x \cdot b=1$. The rank is never more than $n$, and 
thus needs only $\lceil\log n\rceil$ bits to represent. In fact, the rank is at most $O(\log n)$ and thus only needs $O(\log \log n$ bits with high probability. So, we will assume that all three fields are written down in a single word in memory in the form of a triple $x . f=[x . p, x . r, x . b]$. The memory words are of size $2\lceil\log n\rceil+1=O(\log n)$ in this representation. Alternatively, we could have written the algorithm using structs that contain three different words for the three different fields of each node (as in [1]), and obtained the same expected space and work bounds. We picked this formulation because it leads to a concise implementation that is clearer to the reader.

A note about our representation: If the root-bit $x . b$ is set to $1, x$ is a root and thus our implementation disregards the value of the parent field x.p. Notably, our representation is different from the classic representation, where the parent field $x . p=x$ for a root node $x$. This fact is crucial to understanding the pseudo-code.

Concurrency significantly complicates the implementation of the set operations. One complication is that processes can interfere with each other by trying to update the same field at the same time, requiring our algorithms to be robust to such interference. Consider doing unites concurrently. The pseudo code for unite and link is presented in Algorithm 3. In the pseudo-code, $\$$ represents a random bit, i.e. a Bernoulli(1/2). The implementation of the $\operatorname{Find}(x)$ procedure used in the code is discussed in the next subsection.

To do $\operatorname{Unite}(x, y)$, we start as in the sequential case by finding the roots $u$ and $v$ of the trees containing $x$ and $y$, respectively (Line 2). If $u=v$, then $x$ and $y$ are already in the same set and no work needs to be done (Line 3). Otherwise, $x$ and $y$ are in different trees, so we can try to link $u$ and $v$ by doing a CAs to make $v$ the parent of $u$ or vice-versa (Line 4 ). We mark the LINK on Line 4 and subsequent linearization points with an asterisk in the code. (Further explanation of the pivotal LINK procedure is in the next paragraph.) But we must allow for the possibility of the CAs failing. The CAs can fail, for example if it tries to make $v$ the parent of $u$ but in the meantime some other process makes another node the parent of $u$. Notably, it even fails if the other process does the exact same CAs and makes $v$ the parent of $u$. A solution that works in either case is to simply continue walking up the tree from the present $u$ and $v$ (Line 5), until the paths intersect or another attempt at linking is necessary. This method was first proposed by Anderson and Woll [1] and was subsequently used by Jayanti and Tarjan [17].

A key novelty in our algorithm is our linking rule, which we call randomized linking by rank. In the classic linking by rank heuristic [24], each node $x$ has an associated rank field $x . r$, that is an approximation of the height of $x$ in its tree; so, initially all ranks are zero. When two roots $u$ and $v$ are to be linked, the node of larger rank wins, i.e. it becomes the parent of the other node. If the two nodes have equal rank, then the winner is chosen arbitrarily, but its rank is incremented by one to reflect its (possible) increase in height. Anderson and Woll implemented a parallelization of this heuristic, but were unable to get a good work bound [1].

Linking by rank is easy to execute concurrently when the ranks of the nodes $u$ and $v$ in question are different; one simply needs to change the parent of the smaller ranked node. However, when the nodes are of equal rank, two changes have to take place: the parent of one node must be updated, while the rank of the other must also be updated. Making these two changes atomically seems impossible, since the two fields to be changed belong to different nodes, and neither the write nor the CAs primitive can modify two fields in a single step.

Anderson and Woll's implementation handles the equal rank case by always updating the parent pointer first, and rank field second. This implementation allows one process to modify $u_{1} . p$ to $u_{2}$, and another to modify $u_{2} \cdot p$ to $u_{3}$ etc. where all of the $u_{i}$ have the same rank. If all $p$ processes partake in such a sequence of links, the equal rank chain can grow to length $\Omega(p)$. These equal rank chains cause their algorithms to have linear concurrency overhead.

A natural alternative strategy would update the rank before the parent. Unfortunately, this can lead to ranks becoming too large and no longer serving as good proxies for height; which in turn breaks down the efficiency guarantees of linking by rank. Preliminary calculations suggest that this method also leads to concurrency overheads that are at least linear in $p$. Our key insight lies here: while updating parent first or rank first each lead to an algorithm with linear concurrency overhead, we show that picking between the two randomly and either only updating the parent or only incrementing the rank yields an algorithm with at most logarithmic concurrency overhead.

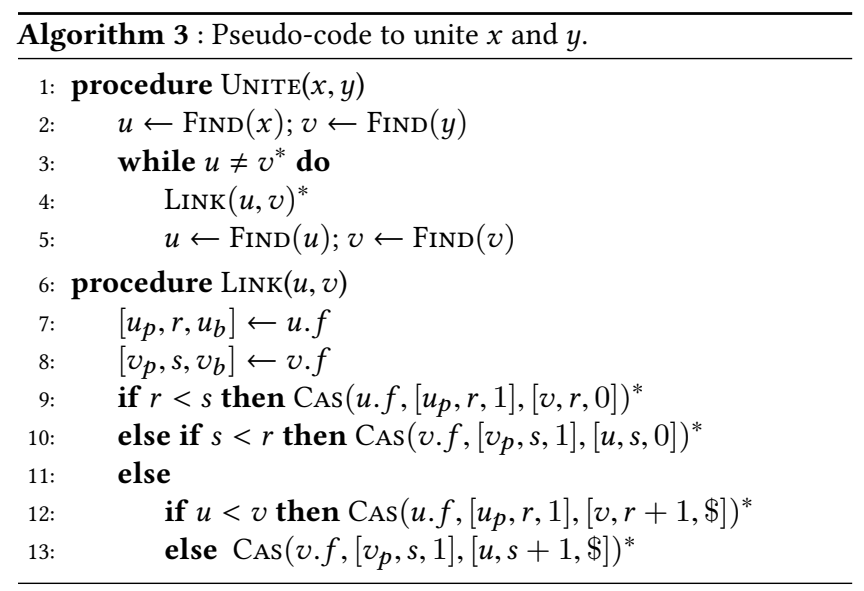

An explanation of our Linking heuristic: Link initally reads the fields of $u$ and $v$ (Lines 7-8). If $u$ 's rank, $r$, is less than $v$ 's rank, $s$, it attempts to change $u$ 's parent to $v$, and $u$ 's root-bit to 0 ("not a root”), while leaving the rank unchanged (Line 9). If $v$ 's rank, $s$, is less than $u$ 's rank, $r$, a symmetric change is attempted (Line 10). The implementation is subtle in the case where $r=s$ : if $u<v$ (check on Line 12 succeeds), the algorithm tries to update $u$ 's parent to $v$ and increment $u$ 's rank, and also set $u$ 's root-bit randomly (Line 12). The previous statement deviates from our intuitive description of the algorithm, so we now explain why the algorithm tries to change both the parent and rank fields. If the update succeeds and the root-bit, $u . b$, gets set to 1 , then $u$ continues to be a root, and thus the parent field is disregarded by the algorithm, so it does not matter that it was updated to $v$. On the other hand, if the update succeeds and root-bit gets set to 0 , then $u$ is no longer a root, and the rank field is thenceforth disregarded by the algorithm, so it does not matter that it was incremented to $r+1$. Line 13 acts symmetrically in the case that $u . r=v . r$ and $v<u$ as opposed to $u<v$. 


\subsection{The Find Procedure}

We present two different implementations of $\operatorname{FinD}(x)$ : naïve and two-try splitting in Algorithm 4. The first procedure, Naïve find simply uses an auxiliary variable $u$ (Line 2 ) that walks up the parent pointers in the tree (Line 4) until it reaches a root (Line 3). FInD $(x)$ returns this node as the representative (Line 5). The linearization point of this procedure is the time at which $u$ is discovered to be a root. The sequence of nodes traversed by $u$ is the find sequence.

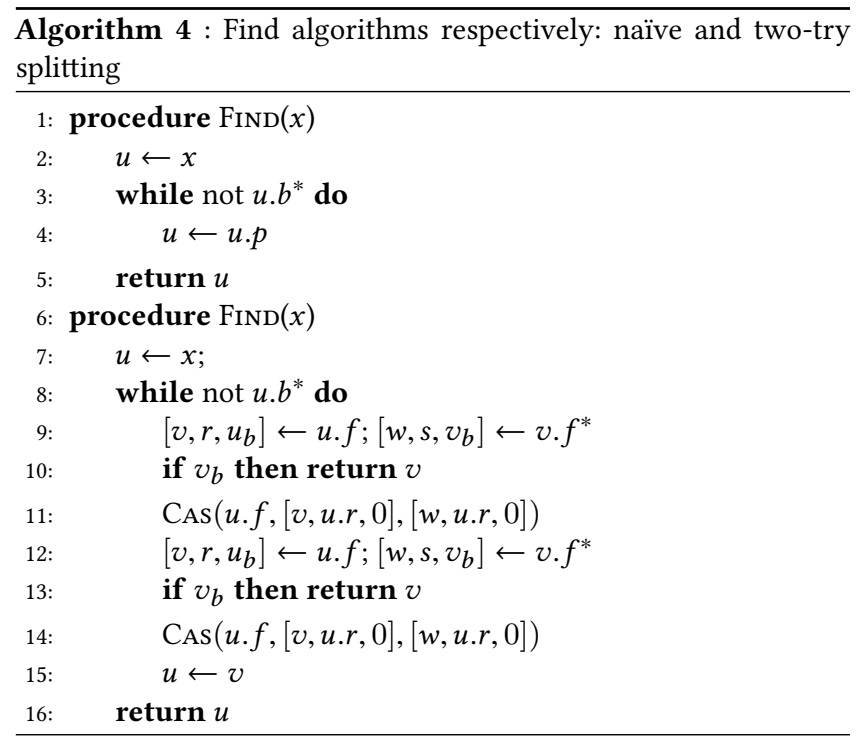

Subsequent finds can be made more efficient if the parents of nodes on the find sequence are updated to be closer to the root. Such changes are said to compact the tree. Splitting is a classical compaction rule that was discovered by Tarjan and van Leeuwen [24], and used by Jayanti and Tarjan in their concurrent algorithm [17]. Rather than using the auxiliary variable $u$ to simply walk up the find sequence, splitting calculates $u$ 's parent $v$ and $v$ 's parent $w$, and tries to improve $u$ 's position in the tree by changing $u$ 's parent to $w$ through a CAs. Find with splitting moves up the find sequence in this way until it has reached a root to return.

Work complexity bounds for splitting are derived through a potential argument. In the argument, a process performing a find operation gains potential each time it successfully improves a node's parent pointer to one closer to the root. Thus, it is important to account for the number of successful improvements in an execution of find in order to calculate the potential difference caused by the find. In the sequential case, all attempts to improve the tree succeed. In the concurrent case however, attempts at improvement can fail if another interfering process successfully changes $u$ 's parent between the time the original process reads $u$ 's parent and attempts its CAS. Jayanti and Tarjan miss some cases of this interference in their complexity analysis. Their bounds increase when interference is properly handled.

In order to overcome this issue, we develop the novel two-try splitting algorithm that tries to improve each node $u$ on the find sequence twice. Although both attempts to improve $u$ may fail in two-try splitting, our analysis shows how the potential decrease caused by interfering processes can be redistributed to account for the work done by all the processes in question if two attempts are made. Unfortunately, the same argument does not hold for the simpler one-try splitting.

An explanation of the two-try splitting find pseudo-code: Find uses an auxiliary variable $u$ to walk up the tree (Line 7). If $u$ is not a root (Line 8), its parent $v$, and $v \cdot p=w$ are read (Line 9). If $v$ is a root, Find has succeeded and can return $v$ (Line 10); otherwise, an improvement is attempted on Line 11. A successful CAS on Line 11 changes only $u$ 's parent, and does not modify the other fields. After a second attempt to improve the same node $u$ (Lines 1214), $u$ is updated to its parent $v$ in order to keep walking up the tree (Line 15). Finally, if $u$ becomes the root (Line 8), it is returned on Line 16. The linearization point of the procedure is the time when the root-bit of the returned node is read, since that is time at which the returned node is surely the root of the tree.

\subsection{Main Theorem}

We now state the main amortized upper bound result about our set union algorithm. We defer the proof of this theorem to the full version of this paper due to the page limit on this extended abstract.

THEOREM 4.1. Given a union-find instance withn nodes on a shared memory asynchronous multiprocessor with p processes:

(1) The expected total work of the set union algorithm over $m$ total unite and find operations is $\Theta(m \cdot(\alpha(n, m /(n p))+\log (1+$ $n p / m)))$.

(2) This total work bound is optimal up to constant factors in the family of symmetric algorithms in the parameter regime $m=$ $\Omega(n)$ and $m=\Omega(p)$ since it matches the lower bound from Theorem 3.7.

(3) The worst-case work for a single operation is $\Theta(\log n)$ in expectation and with probability at least $1-1 / n^{c}$ for any $c>0$.

(4) This worst-case per-operation work bound is optimal in expectation across all algorithms (including symmetric and nonsymmetric) in the parameter regime where $p=n^{\Omega(1)}$ since it matches the lower bound from Theorem 3.4.

\section{REMARKS}

We have designed the first concurrent set union algorithm that has sublinear concurrency overhead against an adversarial scheduler, thereby resolving a 28 year old open question from [1]. The concurrency overhead of our algorithm is at most $\Delta(p)=\log p$. Therefore, the speed-up of our algorithm is the near-linear $p / \log p$. Our algorithm has optimal amortized work in the class of symmetric algorithms, which captures the complexities of all known algorithms for the concurrent set union problem. This is shown by our tight lower bound on the expected amortized work complexities of algorithms in this class. We have also presented the generalized wake up technique and have used to to show that linear speed-up cannot be achieved for set union. We hope that the new techniques introduced in this paper can be extended to better understand concurrency overheads and produce more efficient concurrent data structures. 


\section{ACKNOWLEDGMENTS}

We thank Prasad Jayanti for helpful discussions, and Philipp Woelfel and the PODC reviewers for helpful comments. We also thank the AlgoPARC workshop (funded by NSF grant CCF-1745331) where part of our research was conducted.

\section{REFERENCES}

[1] Richard J. Anderson and Heather Woll. 1991. Wait-free Parallel Algorithms for the Union-Find Problem. In Proceedings of the 23rd Annual ACM Symposium on Theory of Computing, May 5-8, 1991, New Orleans, Louisiana, USA. 370-380. https: //doi.org/10.1145/103418.103458

[2] Richard J. Anderson and Heather Woll. 1991. Wait-free Parallel Algorithms for the Union-Find Problem. In Proceedings of the 23rd Annual ACM Symposium on Theory of Computing, May 5-8, 1991, New Orleans, Louisiana, USA. 370-380. https: //doi.org/10.1145/103418.103458

[3] Vincent Bloemen. 2015. On-The-Fly Parallel Decomposition of Strongly Connected Components. Master's thesis. University of Twente.

[4] Vincent Bloemen, Alfons Laarman, and Jaco van de Pol. 2016. Multi-Core OnThe-Fly SCC Decomposition. In Proceedings of the 21st ACM SIGPLAN symposium on Principles and practice of parallel programming (PPoPP '16). to appear.

[5] Norbert Blum. 1985. On the single-operation worst-case time complexity of the disjoint set union problem. In Annual Symposium on Theoretical Aspects of Computer Science (STACS 1985).

[6] Faith Ellen and Philipp Woelfel. 2013. An Optimal Implementation of Fetchand-Increment. In Proceedings of the 27th International Symposium on Distributed Computing - Volume 8205 (DISC 2013). Springer-Verlag New York, Inc., New York, NY, USA, 284-298. https://doi.org/10.1007/978-3-642-41527-2_20

[7] Wojciech Fraczak, Loukas Georgiadis, Andrew Miller, and Robert Endre Tarjan. 2014. Corrections to "Finding dominators via disjoint set union" [J. Discrete Algorithms 23 (2013) 2-20]. F. Discrete Algorithms 26 (2014), 106-110. https: //doi.org/10.1016/j.jda.2014.01.002

[8] Michael L. Fredman and Michael E. Saks. 1989. The Cell Probe Complexity of Dynamic Data Structures. In Proceedings of the 21st Annual ACM Symposium on Theory of Computing, May 14-17, 1989, Seattle, Washigton, USA. 345-354. https: //doi.org/10.1145/73007.73040

[9] Joel Friedman. 2003. A proof of alon's second eigenvalue conjecture. In Proceedings of the thirty-fifth annual ACM symposium on Theory of computing. ACM, 720-724.

[10] Ashish Goel, Sanjeev Khanna, Daniel H. Larkin, and Robert Endre Tarjan. 2014. Disjoint Set Union with Randomized Linking. In Proceedings of the TwentyFifth Annual ACM-SIAM Symposium on Discrete Algorithms, SODA 2014, Portland, Oregon, USA, January 5-7, 2014. 1005-1017. https://doi.org/10.1137/1. 9781611973402.75

[11] Maurice Herlihy. 1991. Wait-free Synchronization. ACM Trans. Program. Lang. Syst. 13, 1 (Jan. 1991), 124-149. https://doi.org/10.1145/114005.102808

[12] Maurice Herlihy and J. Eliot B. Moss. 1993. Transactional Memory: Architectural Support for Lock-free Data Structures. SIGARCH Comput. Archit. News 21, 2 (May 1993), 289-300. https://doi.org/10.1145/173682.165164

[13] Maurice P. Herlihy and Jeannette M. Wing. 1990. Linearizability: A Correctness Condition for Concurrent Objects. ACM Trans. Program. Lang. Syst. 12, 3 (July 1990), 463-492. https://doi.org/10.1145/78969.78972

[14] Shlomo Hoory, Nathan Linial, and Avi Wigderson. 2006. Expander graphs and their applications. Bull. Amer. Math. Soc. 43, 4 (2006), 439-561.

[15] Prasad Jayanti. 1998. A Time Complexity Lower Bound for Randomized Implementations of Some Shared Objects. In Proceedings of the Seventeenth Annual ACM Symposium on Principles of Distributed Computing (PODC '98). ACM, New York, NY, USA, 201-210. https://doi.org/10.1145/277697.277735

[16] Prasad Jayanti. 2002. F-arrays: Implementation and Applications. In Proceed ings of the Twenty-first Annual Symposium on Principles of Distributed Computing (PODC '02). ACM, New York, NY, USA, 270-279. https://doi.org/10.1145/ 571825.571875

[17] Siddhartha V. Jayanti and Robert E. Tarjan. 2016. A Randomized Concurrent Algorithm for Disjoint Set Union. In Proceedings of the 2016 ACM Symposium on Principles of Distributed Computing (PODC '16). ACM, New York, NY, USA, 75-82. https://doi.org/10.1145/2933057.2933108

[18] Joseph B. Kruskal. 1956. On the Shortest Spanning Subtree of a Graph and the Traveling Salesman Problem. Proc. Amer. Math. Soc. 7, 1 (Feb. 1956), 48-50. http: //www.jstor.org/stable/2033241

[19] Chris Lattner and Vikram Adve. 2002. Automatic Pool Allocation for Disjoint Data Structures. SIGPLAN Not. 38, 2 supplement (June 2002), 13-24. https: //doi.org/10.1145/773039.773041

[20] John Reif. 1985. Depth First Search is Inherently Sequential. Information Processing Letters 20 1, 2 (1985), 229-234.

[21] Robert Sedgewick and Kevin Wayne. 2011. Algorithms, 4th Edition. AddisonWesley. I-XII, 1-955 pages.
[22] Robert Tarjan. 1972. Depth first search and linear graph algorithms. SIAM JOURNAL ON COMPUTING 1, 2 (1972).

[23] Robert Endre Tarjan. 1975. Efficiency of a Good But Not Linear Set Union Algorithm. F. ACM 22, 2 (April 1975), 215-225. https://doi.org/10.1145/321879.321884

[24] Robert E. Tarjan and Jan van Leeuwen. 1984. Worst-case Analysis of Set Union Algorithms. F. ACM 31, 2 (March 1984), 245-281. https://doi.org/10.1145/62. 2160

\section{A SAME SET PSEUDO-CODE}

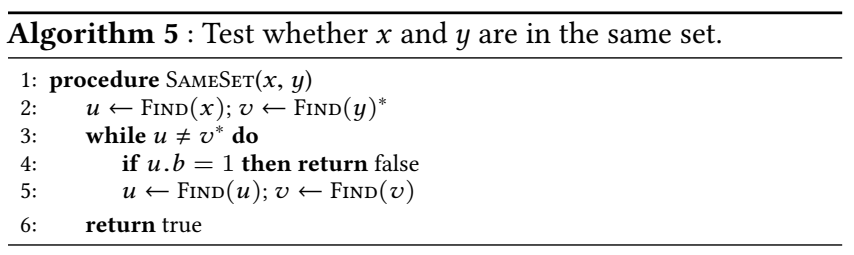

The pseudo-code is an adaptation of Anderson and Woll's same set algorithm [2]. The procedure starts by finding the roots of $x$ and $y$ 's respective trees (Line 2). If the roots are the same (Line 3), then $x$ and $y$ are in the same set and thus true is returned (Line 6). However if they are not the same, then there are two possibilities:

Case 1: Some other process has performed a successful UnITE and thus $u$ is no longer a root (check on Line 4). In this case, the algorithm simply tries again, but starts its finds at the higher nodes $u$ and $v$ (Lines 5).

Case 2: The first root to be discovered, namely $u$, is still a root (check on Line 4). In this case, it can safely be concluded that the root of $y$ 's tree was not $u$ at the linearization point of the $\operatorname{Find}(y)$ on Line 2, since $u$ was a root from the time of $\operatorname{FIND}(x)$ to Line 4 , and $v$ was a (different) root at the time of $\operatorname{Find}(y)$. So, the algorithm returns false on Line 4.

\section{B OTHER AMORTIZED LOWER BOUNDS}

We give amortized lower bounds for counters and ordered collections in this section. We define the data structures and simply state the results that can be shown via reduction to generalized wake up. Due to space constraints in the extended abstract we omit proofs. An $r$-approx-counter is an $r$-additive approximation of a counter.

Theorem B.1 (Counters).

(1) Let $\mathcal{A}$ be any linearizable randomized algorithm for fetchand-inc. For every $m \geq p$, there is a sequence of $m$ operations and a schedule that forces $\mathcal{A}$ to take $\Omega(m \log p)$ steps to perform the operations.

(2) Let $\mathcal{A}$ be any linearizable randomized algorithm for a $p(1-$ $\varepsilon) / 2$-approx-counter, where $\varepsilon>0$ is a constant. For every $m \geq$ $2 p$, there is a sequence of $m$ operations and a schedule that forces $\mathcal{A}$ to take $\Omega(m \log p)$ steps to perform the operations.

Remark. Theorem B.1 shows the step complexity optimality of two algorithms: (1) Ellen and Woelfel's linearizable wait-free fetch-andincrement construction in [6], and (2) Jayanti's counter in [16].

Theorem B.2. Let $\mathcal{A}$ be any linearizable randomized algorithm for an $r$-relaxed Queue, $r$-relaxed Stack, or $r$-relaxed Priority Queue for $r=(1-\varepsilon) p$, where $\varepsilon>0$ is a constant. For every $m \geq p$, there is a set of $m$ REMOVE operations and a schedule that forces $\mathcal{A}$ to take $\Omega(m \log p)$ steps to perform the operations. 